\title{
Evolution of Rotation in Binaries: Physical Processes
}

\author{
Michel Rieutord \\ Observatoire Midi-Pyrénées, 14 avenue Edouard Belin, 31400 Toulouse, \\ France
}

\begin{abstract}
In this review, we describe the physical processes driving the dynamical evolution of binary stars, namely the circularization of the orbit and the synchronization of their spin and orbital rotation. We also discuss the possible role of the elliptic instability which turns out to be an unavoidable ingredient of the evolution of binary stars.
\end{abstract}

\section{Introduction}

The evolution of rotation is usually associated with an evolution of angular momentum; changing the angular momentum of any body requires torques and stars do not escape from this law of physics. In binary stars there is a permanent source of torques: the tides. Hence, understanding the evolution of rotation of stars in a binary system demands the understanding of the action of tides. This compulsory exercise was started more than thirty years ago by Jean-Paul Zahn during his "Thèse d'état", Les marées dans une étoiles double serrée (Zahn 1966). All the concepts needed to understand tides and their actions in the dynamical evolution of binaries are presented in this work.

\subsection{Why Should We Consider the Rotation in Binary Stars ?}

Surely, as in isolated stars, rotation is an important ingredient of evolution through the induced mixing processes: turbulence in stably stratified radiative zones, circulations... All these processes will influence the abundances of elements in the atmospheres or the internal profile of angular velocity, for instance.

However, in binary stars new phenomena appear: tides. They make the orbit evolving, force some mixing processes (through eigenmode resonances for instance) or may even generate instabilities leading, to some turbulence (see below).

These new phenomena need also to be understood if one wishes to decipher the observations of binary stars. In this respect binary stars offer more observables than single stars like the parametres of the orbit, masses of the stars, their radii, etc. If the system has not exchanged mass during evolution and if other parameters like luminosity, surface gravity, abundances can also be determined unambiguously, binary stars offer new constrains on the stars which may be useful for our understanding of stellar evolution. Also, a statistical view of or- 
bital parameters may constrain the dissipative processes at work in these stars (Mathieu et al. 1992).

\subsection{Synchronisation and Circularization : How it Works.}

Let us consider an isolated system made of two stars of mass $\mathrm{M}_{1}, \mathrm{M}_{2}$, of moment of inertia $I_{1}, I_{2}$ and of spin angular velocity $\vec{\Omega}_{1}, \vec{\Omega}_{2}$. The semi-major axis of the orbit is $a$ and the eccentricity $e$. For simplicity we shall assume that the angular momentum vectors are all aligned. Hence, the total (projected) angular momentum of the system, which is conserved during evolution, reads:

$$
L=\frac{M_{1} M_{2}}{M_{1}+M_{2}} a^{2} \Omega_{\text {orb }} \sqrt{1-e^{2}}+I_{1} \Omega_{1}+I_{2} \Omega_{2}
$$

On the other hand, the total energy of the system, namely,

$$
E=-\frac{G M_{1} M_{2}}{2 a}+\frac{1}{2} I_{1} \Omega_{1}^{2}+\frac{1}{2} I_{2} \Omega_{2}^{2}
$$

decreases because of dissipative processes.

To appreciate the natural evolution of such a system, let us consider the even more simplified system where the angular momentum and the energy of the spin of the stars are negligible compared to their orbital equivalent. Using Kepler third law to eliminate the mean angular velocity of the orbital motion $\Omega_{\text {orb }}$, the previous equations lead to

$$
L_{\text {system }} \sim a^{1 / 2} \sqrt{1-e^{2}} \text { and } E_{\text {system }} \sim-\frac{G M_{1} M_{2}}{2 a}
$$

During evolution the system loses energy through dissipative mechanisms, thus $a$ decreases which implies that $e$ also decreases to insure a constant angular momentum. Thus, with time the orbit slowly circularizes.

Once the orbit is circular or nearly circular, the system may continue to evolve if the orbital angular velocity and spin angular velocity are not identical: this is the synchronization process after which the system has reached its minimum mechanical energy state: all the stars rotate at the same rate, i.e. $\Omega_{\text {spin }}=\Omega_{\text {orb }}$ like the moon on its terrestrial orbit.

\subsection{Tides}

In the foregoing section we presented a global view of the evolution of the system, however the way the orbit or the spin change is controlled by the torques applied to the stars. As we said at the beginning, a permanent source of torques is given by the tides which therefore need to be studied. But what is a tide?

The tide is the fluid flow generated by the tidal potential, i.e. the azimuth dependent part of the gravitational potential inside a star. In short, if you sit on a star, it is the forced flow generated by the celestial bodies orbiting around you. If you sit on Earth you feel the tides of the moon and the sun essentially.

Now let us assume that the tidal forcing is mild enough so that the fluid flow obeys linear equations; formally, we may write the system like

$$
\rho \frac{\partial \vec{v}}{\partial t}+\mathcal{L}(\vec{v})=\vec{F}_{T}(\vec{r}) f(t)
$$


where we assume that the tidal force $\vec{F}_{T}(\vec{r}) f(t)$ can be separated into its spatial and temporal dependence. Written in this way we immediately see that if the inertia of the fluid can be neglected (i.e. the term $\rho \frac{\partial \vec{v}}{\partial t}$ ), then the velocity field can be computed with the same temporal dependence as the exciting force. The response is instantaneous. Moreover, if Coriolis acceleration and viscosity are negligible, the only response of the fluid is through a pressure perturbation, i.e. it is purely hydrostatic. This extremely simple, but not unrealistic, case is called the equilibrium tide.

On Earth, this tide is enough to understand the basic properties of terrestrial tides: i.e. that there are two tides a day, that their amplitude is $\sim 50 \mathrm{~cm}$ or that they are stronger at full or new moon; the hydrostatic perturbation describes the famous tidal bulge which is often representing tides in elementary courses. Such a description is appropriate if you sit on the mediterranean beaches or on those of the Pacific ocean; however, if you sit on the Atlantic shore, like here in Cancun, you will easily notice that the tide is much larger than the expected $50 \mathrm{~cm}$. In the Mont Saint-Michel bay it easily reaches 10 meters!

The difference comes from what we neglected: the inertia of the fluid and the ensuing resonance phenomenon. For the tidal wave, whose wavelength is a few thousand kilometers, the Atlantic ocean is a mere puddle five kilometers deep. Surface gravity waves may thus be studied using the shallow water approximation and their speed is given by

$$
V_{\text {wave }}=\sqrt{g h} \simeq 220 \mathrm{~m} / \mathrm{s}
$$

where $g$ is the gravity and $h$ the depth. With a mean width of $5000 \mathrm{~km}$, the Atlantic is crossed twice in 12.6 hours; but the tidal forcing is back after 12.4 hours. Obviously, we are close to a resonance and in this case the equilibrium tide is insufficient to describe the tidal response of the fluid. In this case the tide will be qualified as dynamical.

Quite clearly, the equilibrium tide is much easier to handle than the dynamical one; this is why it was first studied (Zahn 1966); the dynamical tide received first serious considerations by Zahn (1970) and a proper treatment by Zahn (1975).

\subsection{Torques and Tidal Evolution}

In order to compute the dynamical evolution of the system, namely the parameters of the orbit and the spin of the stars it is necessary to evaluate both the torques and the dissipation inside the stars.

The torque suffered by a star results from an unsymmetrical distribution of mass with respect to the tidal potential; mathematically,

$$
\mathcal{T}_{z}=-\int_{(V)}\left(\vec{r} \times \rho \vec{\nabla} \Phi_{T}\right)_{z} d V=-\int_{(V)} \frac{\partial \Phi_{T}}{\partial \varphi} \rho^{\prime} d V
$$

where $\rho^{\prime}$ is the density perturbation generated by the tidal potential $\Phi_{T} ; \varphi$ is the angular azimuthal variable. We see from this expression that torques can only exist if the excitation $\Phi_{T}$ and the response $\rho^{\prime}$ are out of phase (or antiphase). The 
phase lag between these two quantities comes from the dissipative mechanisms at work in the stars and is usually a small but important quantity.

From the expressions of the energy and angular momentum of the orbit, we can derive:

$$
\begin{gathered}
\frac{d \ln a}{d t}=\frac{\dot{E}_{\text {orb }}}{\left|E_{\text {orb }}\right|} \\
\frac{d e^{2}}{d t}=\left|E_{\text {orb }}\right|^{-1}\left(\left(1-e^{2}\right) \dot{E}_{\text {orb }}-\Omega \sqrt{1-e^{2}} \dot{L}_{\text {orb }}\right)
\end{gathered}
$$

where $\dot{E}_{\text {orb }}$ and $\dot{L}_{\text {orb }}$ are respectively the power dissipated in the stars and the torques exerted on the orbit (the parallelism of angular momentum vectors is still assumed).

The foregoing discussion therefore shows that, provided the stars do not change, all the evolution of the system is controlled by the dissipation of energy by the tidal flow.

In the case stellar evolution is important, for instance before or after the main sequence, changes in the inertia momenta will change the spin of the stars and the applied torques.

\section{Dissipation Mechanisms}

\subsection{Viscosity}

The first and most obvious physical mechanism to dissipate mechanical energy is viscosity. However, the viscosity of stellar plasma is far too weak to be efficient and only turbulent viscosity of convection zones can significantly affect the evolution of the system.

But the effectiveness of turbulent viscosity is hampered by the fact that tidal flows are periodic in time: in any region of a convective zone where the lifetime of eddies is longer than the period of the tidal flows, the turbulent eddy viscosity is reduced. The question is, of course, how much it is reduced compared to its usual approximation

$$
\nu_{T}^{0}=\frac{1}{3} V_{\mathrm{turb}} \ell_{\mathrm{turb}}
$$

where $V_{\text {turb }}$ and $\ell_{\text {turb }}$ are respectively the velocity of turbulent eddies and their mean free path.

Presently, two prescriptions coexist: the first by Zahn (1966) says that

$$
\nu_{T}=\nu_{T}^{0} \min \left(1, \frac{V_{\text {turb }} P_{\text {tide }}}{2 \ell_{\text {turb }}}\right)
$$

which means that when the period of tides is shorter than the turnover time of the eddies, the mean-free path of the eddies should be reduced to the distance covered by an eddy during half a period.

The second prescription is originally due to Goldreich \& Keeley (1977) but adapted by Golman \& Mazeh (1991); it says that 


$$
\nu_{T}=\nu_{T}^{0} \min \left(1,\left(\frac{V_{\text {turb }} P_{\text {tide }}}{2 \pi \ell_{\text {turb }}}\right)^{2}\right)
$$

This prescription means that the turbulent viscosity should be such that

$$
\nu_{T}\left(\frac{V_{\text {tide }}}{\ell_{\text {tide }}}\right)^{2}=<\varepsilon>
$$

namely that the dissipation by tidal currents fits the dissipation by the turbulent cascade $\langle\varepsilon\rangle$ (recall that in the Kolmogorov cascade $\langle\varepsilon\rangle \sim V_{\ell}^{3} / \ell$ ).

These two prescriptions yield different exponent in the dependence of the evolution time scale with the period of the system; in principle, they can be discriminated by observations. However, this exercise turns out to be difficult; moreover, using binaries in stellar clusters, Mathieu et al. (1992) found that no reduction of viscosity was necessary to explain observations ! Obviously, more work is needed to clarify this question.

Another viscous damping process was also put forward by Tassoul (1987), namely the dissipation in Ekman boundary layers. However, we have shown that because of the stress-free surfaces of the stars, such layers are rather regions with lower dissipation than the rest of the star (see Rieutord 1992, Rieutord \& Zahn 1997).

\subsection{Radiative Damping}

Another way to dissipate energy of tidal flows is through radiative damping. This mechanism will affect essentially radiative zones; indeed, from the tidal excitation, one needs to generate temperature fluctuations which are dissipated by radiative diffusion.

The most natural way to generate these temperature fluctuations comes from the excitation of gravity modes since, mechanically, they are associated with the buoyancy force. It is therefore quite clear that the dissipation and the ensuing torques will be most important when the forcing frequency is near that of an eigenmode of the star. From this remark, it is also clear that only the dynamical tide will be relevant in this process.

On general grounds we may observe that the tidal forcing is low-frequency and that low-frequency gravity modes are high order modes which can then be described by an asymptotic theory. This was the way chosen by Zahn (1975) and later by Goldreich \& Nicholson (1989).

This mechanism is essentially relevant for early-type stars which own an outer radiative zone. As shown by the previous authors the tidal excitation is most intense near the core-envelope boundary but since gravity waves are only partially reflected at the star surface, they deposit their angular momentum there and these layers are synchronized first. This argument was developed by Golreich \& Nicholson (1989) to explain the higher synchonization rate observed in early-type stars compared to the theoretical predictions of Zahn (1977).

These studies are based on an asymptotic approach and ignore the role of the Coriolis force. This force is, however, unavoidable since the stars are 
always rotating and in most cases the tidal forcing is also in the band of the socalled 'inertial modes' whose restoring force is precisely the Coriolis force. In fact inertial modes always combine with gravity modes as they are also low-frequency modes; both together, they occupy the band $\left[0, \max \left(2 \Omega_{s}, N_{\max }\right)\right]$, where $N$ is the Brunt-Väisälä frequency.

Recently, much progress was achieved in the direction of including the whole spectrum of low-frequency modes in the tidal response of an early-type star. Indeed, Witte \& Savonije (1999a,b,2001) computed numerically the response of a $10 \mathrm{M}_{\odot}$ main sequence star and the ensuing evolution of the system with various inital eccentricities. Their results show many interesting features:

- The efficiency of resonance crossing at decreasing the eccentricity when it is initially small (a few percent).

- A new phenomenon, which they called "resonance locking" and by which two resonant modes, one tending to spin-up the star and the other trying to spin it down, yield equilibrating torques which maintain the two stellar modes close to resonance and therefore force a strong evolution of eccentricity (see figure 3 in Witte \& Savonije 1999b).

The calculations of Witte \& Savonije show that radiative damping is efficient at reducing the eccentricity of the orbit in a fraction of the lifetime of the star: in their examples, the $10 \mathrm{M}_{\odot}$ star has a lifetime of $20 \mathrm{Myr}$; an initial eccentricity of 0.02 is erased in $1 \mathrm{Myr}$, but an initial eccentricity of 0.25 or 0.7 reduces to 0.1 in 3 Myrs; a further evolution apparently operates on a much longer time scale.

Obviously, if the model of Witte \& Savonije could be used as a true system, it would mean that orbits with $e=0.1$ may be as evolved as $e=0$ ones and differ only by initial conditions.

\subsection{A New Possibility: the Elliptic Instability}

Likely, all the mechanisms by which energy can be dissipated have not been examined. We shall now present a new one caused by the elliptic instability and which is potentially a rather strong source of dissipation.

The elliptic instability has been studied mainly in simple geometries and even in these cases it is a difficult problem. For a recent review we refer the reader to the work of Kerswell (2002). Presently, the work closest to astrophysical applications is that of Seyed-Mahmoud et al. (2000) who investigated this instability in an ellipsoidal configuration for the core of the Earth.

The basic result we need from fluid mechanics is the growth rate $\gamma$ of this instability, namely

$$
\gamma \sim \varepsilon \Omega
$$

where $\varepsilon$ is the ellipticity of streamlines and $\Omega$ the rotation rate of the vortex.

Schematically, this instability may be seen as a parametric instability: the solid body rotating fluid feels a perturbation (the ellipticity of the stream lines) with a frequency $2 \Omega$. Such a periodic forcing can destabilize modes at half its frequency namely $\Omega$; this is precisely the frequency of the so-called spin-over modes (or Poincaré modes). Such modes are solid-body rotation around an axis 
in the equatorial plane. When the instability develops and the amplitude gets sufficiently large, the vortex start to precess and is usually completely destroyed as observed in experiments (Malkus 1989). Hence, this instability may be very important as it is able to dissipate the total kinetic energy of the vortex. But let us consider the astrophysical case.

For the sake of simplicity we consider a main sequence star in a binary system with a circular orbit. The tidal perturbation is provided by a pointmass object. The system is not synchronized and therefore $\Omega_{\text {spin }} \neq \Omega_{\text {orb }}$. In a reference frame rotating with the tidal potential, the main sequence star is like a strained vortex rotating with the angular velocity $\Omega_{\text {spin }}-\Omega_{\text {orb }}$ and enduring an elongation $\varepsilon$ of the tidal potential.

In such a case the instability develop on a time scale $\left(\varepsilon\left(\Omega_{\text {spin }}-\Omega_{\text {orb }}\right)\right)^{-1}$. The energy available for dissipation is

$$
E \sim \frac{1}{2} I_{E I Z}\left(\Omega_{\text {spin }}-\Omega_{\text {orb }}\right)^{2}
$$

where $I_{E I Z}$ is the inertia momentum of the zone developing the elliptic instability. In this first attempt, we shall consider a late type stars and restrict, conservatively, the action of this instability to the convection zone so that it does not interfere with the stratification. We note that the spin-over modes which are destabilized are rigid rotations and are thus not affected by the large turbulent viscosity of the fluid.

Hence, the power dissipated is approximately $\frac{\varepsilon}{2} I_{\mathrm{CZ}}\left(\Omega_{\text {spin }}-\Omega_{\mathrm{orb}}\right)^{3}$

With these two quantities, one can estimate the time scale over which synchronization occurs: this is typically the growth of the elliptical instability. To have orders of magnitude, let us take two solar type stars orbiting their center of mass in 10 days and let their spin be twice faster (i.e. $\Omega_{\text {spin }}=2 \Omega_{\text {orb }}$ ). The time scale is then 64 years, thus very short.

As far as circularization is concerned, we need to restrict ourselves to weakly eccentric orbits ( $e \lesssim 0.1$ say). Using the stars to dissipate energy while the angular momentum of the orbit is assumed constant, the reduction of the eccentricity also occurs on a short time scale, namely a thousand of years.

The numbers thus derived are rather robust as they depend on geometric quantities of the system. In view of the observations, which show that they are systems with a 10 days period which are not circularized nor synchronous, we may wonder why the elliptic instability is not so efficient in binary stars. The answer is likely in the much more complex setup yielded by stars compared to their equivalent in the laboratory. In stars, there are stratification, magnetic fields, time variable ellipticity (on eccentric orbits), rotation etc... All these effects have been explored but most of the time using systems which are not quite similar to a star. Nevertheless, we shall list them and speculate about their effects in a stellar situation.

- Rotation of the frame associated with the orbital motion influences the elliptic instability through the Coriolis force. Craik (1989) has shown, but using an unbounded strained flow, that rotation is either stabilizing or destabilizing. It is stabilizing if, for instance, in an inertial frame, the star does not rotate. 
- Stratification: Similarly to rotation, stratification does not act in a unique sense; using the context of an elliptical cylinder Kerswell (1993) has shown that stratification is stabilizing for polar regions of a star but destabilizing for equatorial regions.

- Magnetic fields were found invariably stabilizing (see Kerswell 1994).

- Time-periodic ellipticity is likely destabilizing through parametric instabilities. However, the only studied cases are those of an unbounded strained fluid (Kerswell 2002).

- Nonlinear effects are the most difficult to appreciate. Experiments have shown that the nonlinear development is violent (Aldridge et al. 1997) and rapidly leads to a turbulent state (Malkus 1989). In fact, it seems that the saturated state exists only in a very narrow range of parameters and beyond this range secondary instabilities due to triadic interactions of inertial modes lead to small scale motions and a turbulent state (Mason \& Kerswell 1999).

Globally, the way the instability saturates may be thought as a change of the spin axis of the fluid so as to reduce the ellipticity of the streamlines. In stars such an effect is possible if the spin axis of stars is inclined in the plane normal to the orbital plane and passing through the centres of mass. This shows that the apparently inocuous hypothesis of angular momentum vectors alignment may be crucial to the instability. It may also be an observational signature. Indeed, we noticed that the turbulent viscosity of convection zone could hardly inhibit the resonance of spin-over modes; however, it can easily suppress secondaries instabilities which, in laboratory experiments lead to a turbulent state. It may well be that the saturated state is difficult to obtain in the laboratory but much more easily in stars.

The foregoing discussion shows that many points of the elliptic instability in the stellar context remain in the shadows; but because of its great potential dissipative power, this instability deserves more study.

\section{Concluding Remarks}

Born together, stars of a binary system share the same age and the same initial metallicity. In most favourable cases, their mass, radii, rotation rates can be determined. These are of course very interesting pieces for the puzzle of stellar evolution but there is a price to pay for these additional informations: the two stars interact during their whole lifetime. In its simplest form this interaction is of gravitational origin and generates tides. As we have shown, tides generate various fluid flows giving rise to transport processes which may be observationnally constrained if a comparison with analogous isolated stars is possible.

The foregoing presentation which sketched out all the known mechanisms by which energy is dissipated also shows that the situation is not simple. Various processes can dissipate energy and we suggest that among them the elliptic instability plays a non negligible part. Quite surprisingly, this instability has been 
overlooked until now. Despite its strength, shown by laboratory experiments, observations do not show, yet, an evidence of this instability. In the list of the mechanisms which may inhibit this instability, we noticed the misalignement of rotation axis. As such a misalignement may also result from a saturated state of this instability and can be an observational signature, it deserves some study.

To conclude this contribution let us point out some directions of research:

- On the theoretical side more work is obviously needed on the effects of the elliptic instability.

- On models, the integration of stellar evolution combined with dynamical (tidal) evolution following up the works of Claret \& Cunha (1997) and Witte \& Savonije (1999b) will be useful to understand, for instance, the statistical properties of eccentricities as a function of periods and ages, or the relative importance of pre-main sequence, main sequence, post-main sequence phases.

- On the observational side, much data are needed. First, the elements describing the dynamics are very much desired: these are the elements of the orbit $(a, e, i, \Omega, \omega)$, the masses $\mathrm{M}_{1}, \mathrm{M}_{2}$, the spins $\vec{\Omega}_{1}, \vec{\Omega}_{2}$ and their variations with time. For instance, the motion of the apsidal line $\dot{\omega}$ is a quantity constraining the mass distribution of the stars and therefore their internal rotation. It is clear that such data will require a lot of efforts but they will help us much in our understanding of stellar evolution. A pulsar like J0045-7319, which travels around an early-type star on a highly eccentric orbit, offers a good step in this direction (Lai et al. 1995).

\section{References}

Aldridge K., Seyed-Mahmoud B., Henderson G. \& van Wijngaarden W., 1997, Phys. Earth Plan. Int. 103, 365

Claret A. \& Cunha N., 1997, A\&A318, 187

Craik A., 1989, J. Fluid Mech. 198, 275

Goldreich, P. \& Keeley, D., 1977, ApJ212, 243

Kerswell, R., 1993, Geophys. Astrophys. Fluid Dyn. 71, 105

Kerswell, R., 1994, J. Fluid Mech. 274, 219

Kerswell, R., 2002, Ann. Rev. Fluid Mech. 34, 83

Lai D., Bildsten L. \& Kaspi V., 1995, ApJ452, 819

Malkus W., 1989, Geophys. Astrophys. Fluid Dyn. 48, 123

Mason D. \& Kerswell R., 1999, J. Fluid Mech. 396, 73

Mathieu R. D., Duquennoy A., Latham D. W., Mayor M., Mermilliod T., Mazeh, J. C., 1992, Binaries as Tracers of Stellar Formation. Proceedings of a Workshop held in Bettmeralp, Switzerland, Sept. 1991, Edts A. Duquennoy, M. Mayor, p. 278

Rieutord, M., 1992, A\&A259, 581

Rieutord, M. \& Zahn, J.-P., 1997, ApJ474, 760

Seyed-Mahmoud B., Henderson G. \& Aldridge K.D., 2000, Phys. Earth Plan. Int. 117, 51

Tassoul, J.-L., 1987, ApJ322, 856 
Witte, M. \& Savonije, G., 1999a, A\&A341, 842

Witte, M. \& Savonije, G., 1999b, A\&A350, 129

Witte, M. \& Savonije, G., 2001, A\&A366, 840

Zahn, J.-P., 1966, Ann. Astrophys., 29, 313, 489, 565.

Zahn, J.-P., 1970, A\&A4, 452

Zahn, J.-P., 1975, A\&A41, 329 